\title{
Research on the Relative Positions-Constrained Pattern Matching Method for Underwater Gravity-Aided Inertial Navigation
}

\author{
Lin Wu, Hubiao Wang, Hua Chai, Houtse Hsu and Yong Wang
}

(State Key Laboratory of Geodesy and Earth's Dynamics, Institute of Geodesy and Geophysics, Chinese Academy of Sciences, Wuhan 430077, P.R. China)

(E-mail: ywang@whigg.ac.cn)

\begin{abstract}
A Relative Positions-Constrained pattern Matching (RPCM) method for underwater gravityaided inertial navigation is presented in this paper. In this method the gravity patterns are constructed based on the relative positions of points in a trajectory, which are calculated by Inertial Navigation System (INS) indications. In these patterns the accumulated errors of INS indicated positions are cancelled and removed. Thus the new constructed gravity patterns are more accurate and reliable while the process of matching can be constrained, and the probability of mismatching also can be reduced. Two gravity anomaly maps in the South China Sea were chosen to construct a simulation test. Simulation results show that with this RPCM method, the shape of the trajectory in gravity-aided navigation is not as restricted as that in traditional Terrain Contour Matching (TERCOM) algorithms. Moreover, the performance included matching success rates and position accuracies are highly improved in the RPCM method, especially for the trajectories that are not in straight lines. Thus the proposed method is effective and suitable for practical navigation.
\end{abstract}

\section{KEY WORDS}

$\begin{array}{llll}\text { 1. Underwater passive navigation. } & \text { 2. INS. 3. Gravity-aided navigation. } \quad \text { 4. Pattern matching }\end{array}$

Submitted: 1 February 2014. Accepted: 5 March 2015. First published online: 26 March 2015.

1. INTRODUCTION. At the moment, research and development of Autonomous Underwater Vehicles (AUVs) has become of special strategic and economic significance. During extended AUV missions, additional aided navigation methods are needed to correct the navigation errors of Inertial Navigation Systems (INS), which increase with time. At present, radar, laser and sonar are deployed on AUVs to establish the underwater navigation system. However, similar to radar navigation close to the surface, laser or active sonar transmission (underwater terrain could be measured for aided navigation) can affect the covertness of AUVs. Gravimetry is not emanative, and not easily detected and interfered with. Gravity-aided navigation is stable and 


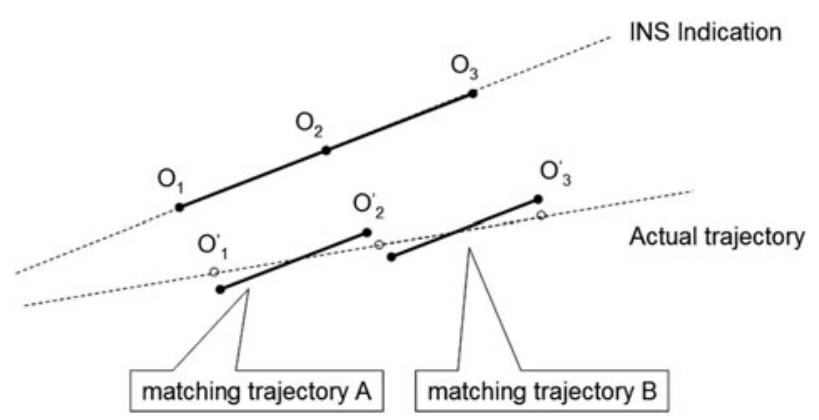

Figure 1. Principle of the TERCOM matching algorithm (Wang et al., 2012).

passive and thus is appropriate for covert underwater navigation. In the past few years, gravity/gravity gradient-aided underwater passive navigation systems have been established on AUVs or submarines to limit INS error accumulation (Affleck and Jircitano, 1990; Goldstein and Brett, 1998; Hays, 2002; Jircitano et al., 1990; Moryl et al., 1998; Rice et al., 2000; Rice et al., 2004; Vajda and Zorn, 1998; Wang and Bian, 2008). Gravimeters and gradiometers were adopted to compensate the INS (Jekeli, 2006; Moody and Paik, 2004). In addition, gravity and gravity gradient have been used for underwater object detection which is a significant subject in the field of underwater navigation (Wu and Tian, 2010; Wu et al., 2010b; Wu et al., 2013).

Researching a precise location algorithm is one of the most important key technologies of underwater navigation systems. Several methods have been developed for gravity matching navigation. Kalman filters were designed to estimate the state parameters of gravity/gravity gradient/geomagnetic aided navigation systems (Wu et al., 2007; Wu et al., 2010a; Wu et al., 2011; Xu, 2005; Zhang et al., 2004b; Zheng et al., 2013). The Iterated Closest Contour Point (ICCP) algorithm was constructed for vehicle localisation in gravity maps (Bishop, 2002; Deng et al., 2010; Kamgar-Parsi and Kamgar-Parsi, 1999). The Terrain Contour Matching (TERCOM) algorithm was explored for terrain matching-aided navigation at first (Golden, 1980; Siouris, 2004), but nowadays the TERCOM algorithm is widely employed for gravity matching navigation. With the TERCOM algorithm, the trajectory with a real-time sequence of gravity measurements is taken to make correlation analysis with the stored gravity maps. Then the optimal matching trajectories are obtained and used to correct the INS (Figure 1).

Conventional TERCOM methods based on pattern or image matching are widely used in terrain-aided navigation. Remote sensing devices such as active sonar, radar, and Charge-Coupled Device (CCD) cameras can be utilised to look around and typically provide many simultaneous measurements, either a two dimensional (2D) image or a one-dimensional (1D) profile which can be used for matching (Cowie et al., 2008; Zhang et al., 2004a). In contrast, a challenging aspect of gravity pattern matching is that nowadays gravimeters can measure the field only in-place, and thus provide only one measurement at a time. Therefore, the traditional 2D pattern or image matching-based TERCOM methods cannot be employed without modification and a novel pattern matching method for gravity aided navigation needs to be explored.

With the original TERCOM algorithm, after passage the gravity measurements over a period of time were collected, then the gravity patterns were constructed based on the 
gravity measurements and the absolute INS indicated positions of points in the trajectory. The gravity patterns in straight lines were taken into the correlation calculations with the gravity maps in the area, so the matching trajectories which were parallel to the INS trajectories could be obtained. The original TERCOM algorithm can work stably and effectively as long as the INS quality is good enough. However, the vehicle must move with constant speed along a straight line (the trajectory is not strictly required to be parallel to one of the database axis directions) during each TERCOM update period (Yoo et al., 2012). So only limited manoeuvring in operation is allowed by the original TERCOM algorithm. More importantly, when the INSindicated positions diverge from the actual trajectory or direction due to the accumulated position errors, huge position errors and even false navigation results may be produced. Apparently, the conventional TERCOM algorithm cannot meet the needs of various nonlinear trajectories in practical navigation. Some modified TERCOM algorithms were developed in which translation and rotation were employed to improve the matching trajectories, but their performance in complicated curving trajectories which are common in practice was not impressive (Wang et al., 2010; Wang et al., 2012; Yoo et al., 2012). An improved TERCOM positioning method using relative position offsets from the INS was proposed for terrain-aided AUV navigation and obtained better performance (Ånonsen, 2005; 2010).

In this paper, a Relative Positions-Constrained pattern Matching (RPCM) method for underwater gravity aided inertial navigation is presented, which is an improved form of TERCOM. In this method the gravity patterns are constructed based on the relative positions of points in a trajectory, which are calculated by INS indications. Then the gravity patterns are taken into the correlation calculation with the stored gravity maps. The accumulated errors of INS indicated positions are cancelled in the relative positions and gravity patterns. Therefore, these new constructed gravity patterns are more accurate and reliable so the process of matching can be constrained; the probability of mismatching can also be reduced. The principle of the RPCM method is described in Section 2. A simulation analysis was performed in two gravity maps from different areas located in the South China Sea to analyse and verify the performance of the proposed RPCM method in Section 3. Finally, Section 4 gives conclusions.

\section{THE RELATIVE POSITIONS-CONSTRAINED GRAVITY PATTERN} MATCHING METHOD. When the AUV is in motion, gravity measurements $g_{i}$ $(i=1,2,3, \ldots)$ at the points $p_{i}\left(x_{i}, y_{i}\right)(i=1,2,3, \ldots)$ along the trajectory can be obtained with the on board gravimeter. Here $p_{i}\left(x_{i}, y_{i}\right)$ are the actual positions of the $\mathrm{AUV}$, and the corresponding INS-indicated positions are $p_{i}^{I N S}\left(x_{i}^{I N S}, y_{i}^{I N S}\right)$. Highprecision INS can give continuous accurate positions to begin with, but the accumulated position errors are still unacceptable after a long period underway. Certainly, there are drift errors between $p_{i}\left(x_{i}, y_{i}\right)$ and $p_{i}^{I N S}\left(x_{i}^{I N S}, y_{i}^{I N S}\right)$. The position error of INS increases slowly with time, but does not increase suddenly and rapidly in a short period. So in a period of time the relative positions between $p_{i}^{I N S}$ and $p_{j}^{I N S}$ can be regarded as accurate and reliable. For example, after 20 hours' sailing the positions indicated by the INS with $0.01 \mathrm{deg} / \mathrm{hr}$ gyroscope bias $\left(p_{i}^{I N S}\left(x_{i}^{I N S}, y_{i}^{I N S}\right)\right)$ may be wide apart from the actual positions $\left(p_{i}\left(x_{i}, y_{i}\right)\right)$, but the relative positions between two points just passed in a few minutes can be more accurate and reliable. 


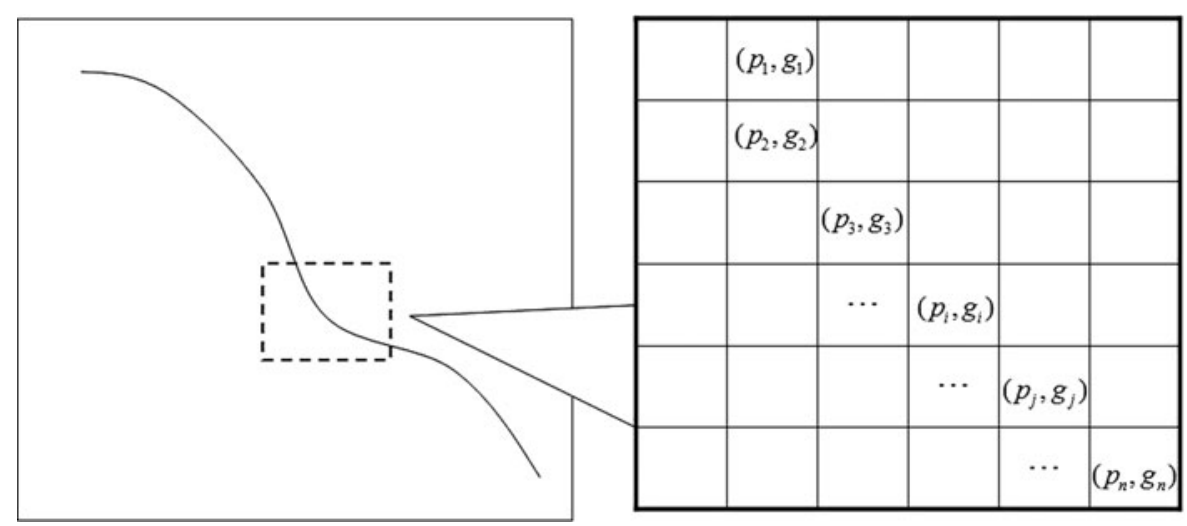

Figure 2. Construction of gravity pattern/image $S$.

Consequently, the relative positions can be found as follows:

$$
\left\{\begin{aligned}
\Delta x_{i j}=x_{j}-x_{i} & \approx x_{j}^{I N S}-x_{i}^{I N S} \\
\Delta y_{i j}=y_{j}-y_{i} & \approx y_{j}^{I N S}-y_{i}^{I N S}
\end{aligned}\right.
$$

Based on this assumption, after the passage the gravity pattern/image $S$ in a period of time can be constructed, as can be seen in Figure 2. In this image $S$, the relative positions $\left(\Delta x_{i j}, \Delta y_{i j}\right)$ between $p_{i}$ and $p_{j}$ are calculated from INS indicated positions with Equation (1), although the actual values of $\left(x_{i}, y_{i}\right)$ or $\left(x_{j}, y_{j}\right)$ themselves are unknown. From Equation (1) it can be seen that the accumulated errors of INS indicated positions are cancelled in the relative positions $\left(\Delta x_{i j}, \Delta y_{i j}\right)$. Also, in this image the positions without measurements are signed with invalid data that would not be used in the following correlation calculation.

The correlation coefficients between gravity map $P$ and the gravity pattern/image $S$ which can be completed only after the passage would be calculated as follows:

$$
\operatorname{COEFS}=\operatorname{COR}\left(S_{m^{*} n}, P_{M^{*} N}\right)
$$

where $S$ is a $m^{*} n$ matrix, $P$ is a $M^{*} N$ matrix, so the size of correlation coefficients matrix COEFS is $(M-m+1)^{*}(N-n+1)$. COR is the correlation algorithm, Mean Absolute Difference algorithm (MAD) and Mean Square Difference algorithm (MSD) can be chosen as the algorithm. The MAD and MSD algorithms have proven to be an effective correlation scheme and their definitions are as follows:

$$
\begin{aligned}
& M A D(x, y)=\frac{1}{M^{*} N} \sum_{u=0}^{M-1} \sum_{v=0}^{N-1}|S(u, v)-P(x+u, y+v)| \\
& M S D(x, y)=\frac{1}{M^{*} N} \sum_{u=0}^{M-1} \sum_{v=0}^{N-1}[S(u, v)-P(x+u, y+v)]^{2}
\end{aligned}
$$

The minimum value of coefficients can be found:

$$
\text { min_coefs }=\min (\text { COEFS })
$$




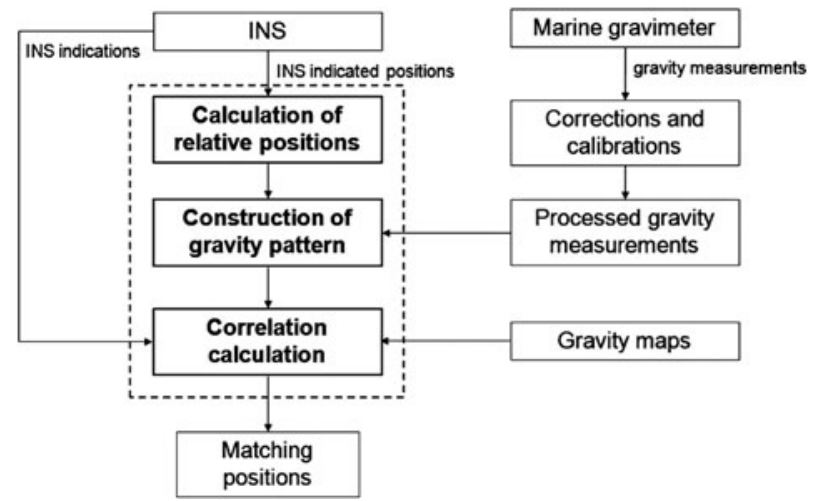

Figure 3. Flow chart of relative positions-constrained gravity pattern matching (RPCM) method.

So the minimum value of coefficients identifies the matching position in the gravity map which is considered to be the optimal matching position.

From the above it can be seen that the RPCM method here and the TERCOM method are both positioning methods based on a correlation calculation with the stored maps. In our proposed method the gravity patterns are constructed based on the relative positions of points in the trajectory, which are calculated by INS indicated positions. The accumulated errors of INS indicated positions are cancelled in the relative positions and gravity patterns, so the gravity patterns are in better agreement with the various actual trajectories. Therefore, these new constructed gravity patterns are more accurate and reliable so the process of matching can be constrained, and higher position accuracy may be achieved by the RPCM method in underwater gravity aided inertial navigation. Actually, although the shapes of trajectories can be recovered better by gravity patterns with the RPCM method, essentially it is just a positioning method in which mostly only position errors but not velocity or attitude errors were included and could be reduced. The effect of attitude or velocity errors that cannot be corrected in the TERCOM method also cannot currently be entirely cancelled by using the proposed method.

The flow chart of the RPCM method is presented in Figure 3.

From this figure it can be found that the proposed RPCM method for underwater gravity-aided inertial navigation can be described as follows.

Firstly, the relative positions of the points in a trajectory are calculated from the INS indicated positions. The relative positions are prepared to construct the new gravity patterns in which the accumulated position errors of INS are cancelled. They are different to the gravity profiles in the traditional TERCOM algorithm that are formed from absolute INS indicated positions.

Secondly, gravity patterns in a period of time are constructed with the calculated relative positions and the processed gravity measurements from the gravimeter. In practice, the influence of temperature, sea conditions and instruments should be considered such that the gravity measurements from the marine gravimeter cannot be used directly for gravity-aided navigation, so several corrections and calibrations must be done with the data. After the Eötvös correction (The Eötvös effect is the change in perceived gravitational force caused by the change in centrifugal acceleration resulting 

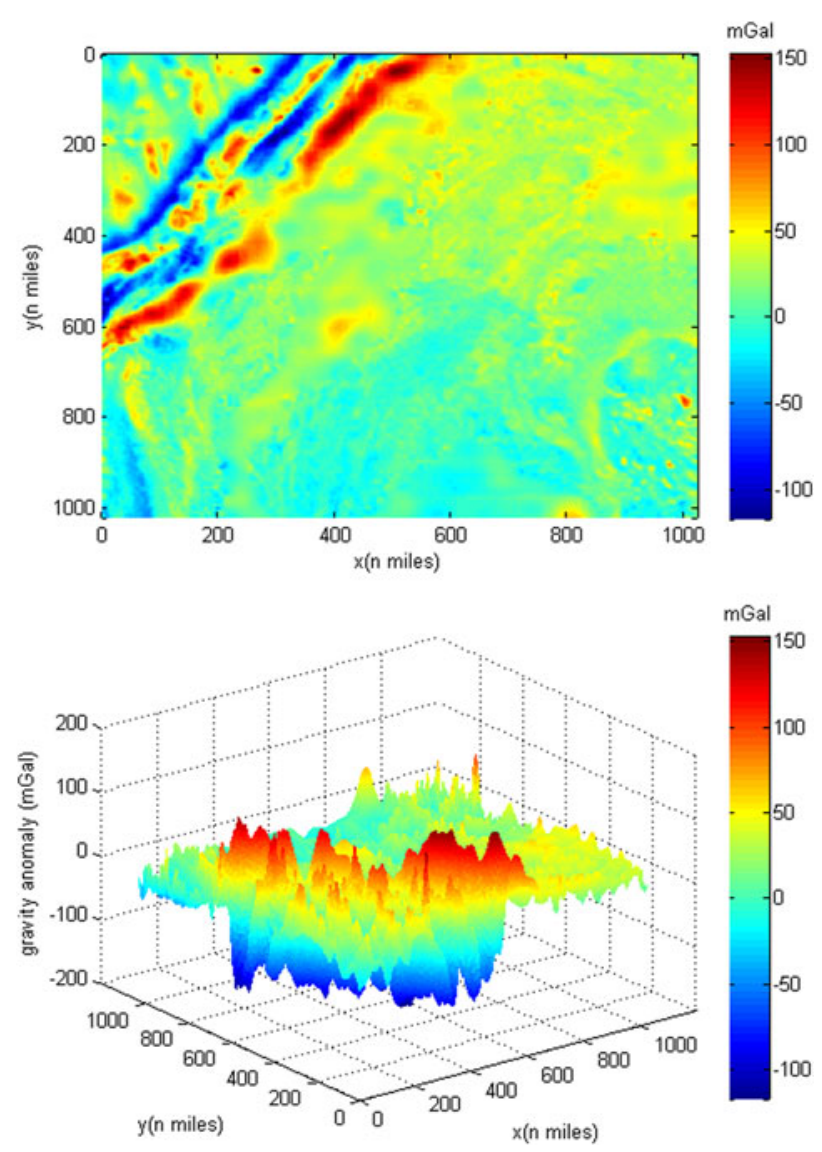

Figure 4. Gravity map $P_{1}$ located in South China Sea.

from eastbound or westbound velocity) and some processing including gravity base point comparison, gravimeter hysteresis effect correction, gravimeter zero point draft correction and vehicle's draft of water correction et al, the processed gravity data which fits the requirement of gravity aided navigation is ready for use.

Then the correlation coefficients between gravity maps and the constructed gravity patterns are calculated. Finally, the extreme value of coefficients identifies the optimal matching positions in the gravity maps.

3. SIMULATION RESULTS AND DISCUSSION. A simulation was performed to verify the proposed RPCM method performance. A gravity anomaly map $P_{1}$ which is located in the South China Sea area was chosen for simulation. The $2 \mathrm{D}$ and $3 \mathrm{D}$ views of the map are demonstrated in Figure 4. The size of map is $1024 \times 1024$ nautical miles $(\mathrm{nm})$ and the resolution is $2 \mathrm{~nm}$ in both $x$ and $y$ directions. The gravity values in the map are gravity anomalies, and their statistical values are given in Table 1, where STD is standard deviation and RMS is root-mean-square.

3.1. Individual trajectory test. A trajectory with the gravity measurements on it is illustrated in Figure 5. In this test the actual trajectory was designed as a slight curved 
Table 1. Statistical Values of Gravity Anomalies in gravity map $P_{1}$ (unit: $\mathrm{mGal}$ ).

\begin{tabular}{lllll}
\hline Maximum & Minimum & Mean & STD & RMS \\
\hline 152.62 & -117.40 & 16.78 & 28.76 & 33.29 \\
\hline
\end{tabular}

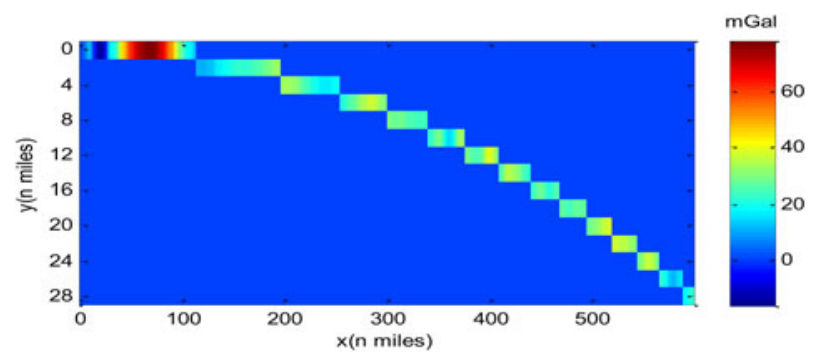

Figure 5. A trajectory and the gravity pattern/image $S$.

line as this is common in practice. During the period of this trajectory, the INS drift was only presented in the $y$ direction that led to the accumulated position errors just in this direction. As a result the INS-indicated positions were in a rectilinear trajectory. As the INS-indicated positions were in a rectilinear trajectory, the TERCOM method can be employed conveniently to make the correlation calculation and obtain a matching trajectory that can be taken to compare with the RPCM method. The curvature of the actual trajectories is much smaller as can be seen in Figure 6(a) than it looks in Figures 5 and 6(b), because Figures 5 and 6(b) are partially zoomed in and stretched in the north-south direction for the effect of display (the size of Figure 5 is $600 \times 30 \mathrm{~nm}$, and Figure $6(\mathrm{~b})$ is nearly $800 \times 160 \mathrm{~nm}$ ). Here the gyroscope bias of the INS is $0.01 \mathrm{deg} / \mathrm{hr}$, the velocity component in the $x$ direction is 2 knots every sample time. A noise model was designed based on the data from a true gravity sensor so that measurement noises are added in the gravity measurements. The MSD algorithm was chosen for the correlation calculation (COR). Compared with TERCOM, in the RPCM method the gravity pattern/image $S$ is constructed based on the relative positions as shown in Figure 5. The accumulated errors of INS indicated positions are cancelled in the relative positions and gravity patterns. So the positions of points in a trajectory can be recovered better with the RPCM method even though the actual effects of attitude errors were not cancelled out in this solution as in the TERCOM method.

In Figure 6 the one-time matching results of an individual trajectory test of TERCOM and RPCM position solutions shows that not only the shape but also the matching positions of the trajectory by RPCM are in better agreement with the actual trajectory. Obviously a higher position accuracy is achieved by the proposed RPCM method. In this test the size of gravity pattern/image $S$ is $300 \times 15$ grids and the size of gravity map $P_{1}$ is $512 \times 512$ grids $(2 \mathrm{~nm} / \mathrm{grid})$, so the correlation coefficients matrix COEFS is a $213 \times 498$ matrix - an image of it is presented in Figure 7. The minimum value of coefficients is 0.6438 that identifies the optimal matching position in the gravity map. 

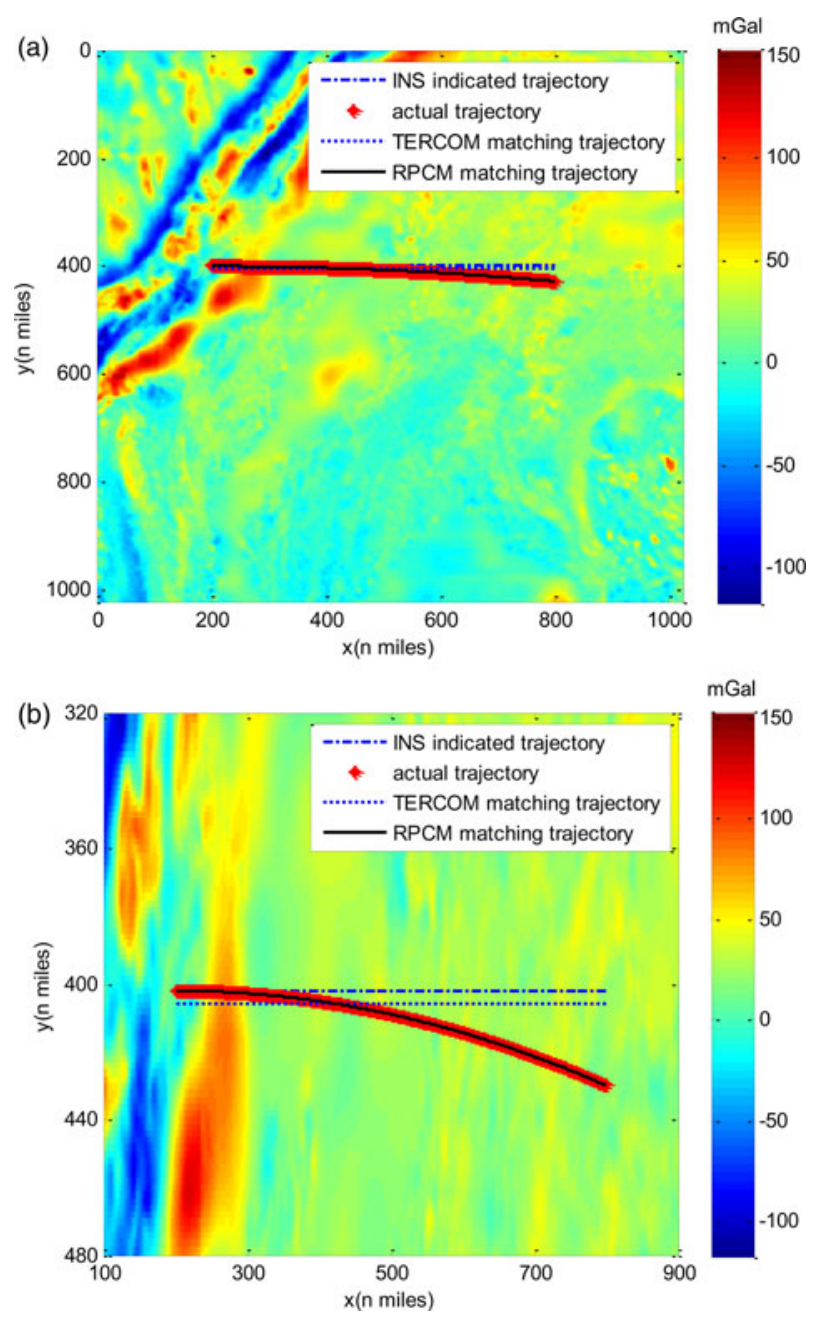

Figure 6. INS indicated and matching trajectories (a) Trajectories in the map $P_{1}$; (b) Partial enlarged drawing.

3.2. Sets of trajectories test. Six sets of trajectories were simulated to test the two positioning methods: RPCM and TERCOM. In every set there are 100 trajectories of curved lines that have the same shape but different stochastic start points. From set No. 1 to 6 the degree of curvature of trajectories in every set increases. Here, the MAD and MSD algorithms were chosen for the correlation calculation $(C O R)$, also the same level of noise as in true measurement data were considered in the gravity measurements. The gyroscope bias of the INS and the vehicle speed component in the westeast direction are the same as the test in Figure 6. A successful matching is defined as that where the position error is smaller than a threshold. Here the matching success rate was calculated when the threshold was assigned as $1.414 \mathrm{~nm}$. After the test matching, success rates and average position accuracies of RPCM and 


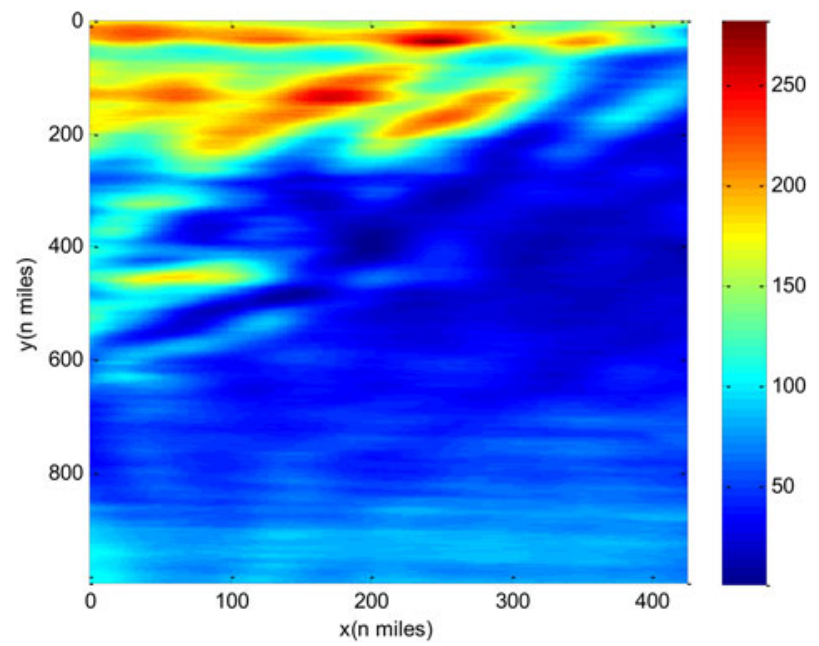

Figure 7. An image of correlation coefficients matrix COEFS.

Table 2. Matching Success Rates and Average Position Accuracies of RPCM and TERCOM Methods (MAD).

\begin{tabular}{lccccc}
\hline & \multicolumn{2}{c}{ RPCM } & & \multicolumn{2}{c}{ TERCOM } \\
\cline { 2 - 3 } \cline { 5 - 6 } Set No. & $\begin{array}{l}\text { Matching success } \\
\text { rate }(* 100 \%)\end{array}$ & $\begin{array}{l}\text { Average position } \\
\text { accuracy (n mile) }\end{array}$ & & $\begin{array}{l}\text { Matching success } \\
\text { rate }(* 100 \%)\end{array}$ & $\begin{array}{l}\text { Average position } \\
\text { accuracy (n mile) }\end{array}$ \\
\hline 1 & $0 \cdot 9$ & $0 \cdot 20$ & & $0 \cdot 3$ & $2 \cdot 34$ \\
2 & $0 \cdot 96$ & $0 \cdot 08$ & & $0 \cdot 34$ & $2 \cdot 38$ \\
3 & $0 \cdot 94$ & $0 \cdot 12$ & & $0 \cdot 22$ & $2 \cdot 31$ \\
4 & $0 \cdot 96$ & $0 \cdot 10$ & & $0 \cdot 28$ & $1 \cdot 97$ \\
5 & $0 \cdot 98$ & $0 \cdot 04$ & & $0 \cdot 3$ & $2 \cdot 26$ \\
6 & $0 \cdot 98$ & $0 \cdot 04$ & & $0 \cdot 22$ & $19 \cdot 46$ \\
\hline
\end{tabular}

TERCOM can be calculated for every set of trajectories. The statistical results are presented in Tables 2 and 3.

It can be seen from Tables 2 and 3 that, from set No. 1 to 6 the matching success rates of TERCOM decrease rapidly, they are less than $34 \%$ for MAD and no more than $28 \%$ for MSD all the time. Meanwhile the average position accuracies of TERCOM get worse and worse, even the best accuracy is $1.97 \mathrm{~nm}$ for MAD and 2.38 nm for MSD.

However, in strong contrast the matching success rates and average position accuracies of the RPCM method perform excellently and remain stable throughout. The matching success rates are higher than $90 \%$ for MAD and higher than $96 \%$ for MSD, the average position accuracies are better than $0 \cdot 20 \mathrm{~nm}$ for MAD and better than $0.08 \mathrm{~nm}$ for MSD during this simulation.

It can also be seen from the curves of matching success rates and average position accuracies in Figure 8 that distinctly improved matching success rates and position accuracies can be gained by this proposed RPCM method. In particular, when the degrees of curvature of trajectories are bigger, the advantages of the RPCM method 
Table 3. Matching Success Rates and Average Position Accuracies of RPCM and TERCOM Methods (MSD).

\begin{tabular}{|c|c|c|c|c|}
\hline \multirow[b]{2}{*}{ Set No. } & \multicolumn{2}{|c|}{ RPCM } & \multicolumn{2}{|c|}{ TERCOM } \\
\hline & $\begin{array}{l}\text { Matching success } \\
\text { rate }\left({ }^{*} 100 \%\right)\end{array}$ & $\begin{array}{l}\text { Average position } \\
\text { accuracy (n mile) }\end{array}$ & $\begin{array}{l}\text { Matching success } \\
\text { rate }(* 100 \%)\end{array}$ & $\begin{array}{l}\text { Average position } \\
\text { accuracy ( } \mathrm{n} \text { mile }\end{array}$ \\
\hline 1 & 0.98 & $0 \cdot 04$ & $0 \cdot 18$ & $2 \cdot 38$ \\
\hline 2 & 0.98 & 0.04 & $0 \cdot 18$ & $2 \cdot 93$ \\
\hline 3 & 0.98 & $0 \cdot 04$ & $0 \cdot 2$ & $3 \cdot 21$ \\
\hline 4 & 0.98 & $0 \cdot 04$ & $0 \cdot 28$ & $6 \cdot 13$ \\
\hline 5 & 0.96 & 0.08 & $0 \cdot 2$ & $12 \cdot 26$ \\
\hline 6 & 0.98 & $0 \cdot 04$ & $0 \cdot 14$ & 18.03 \\
\hline
\end{tabular}
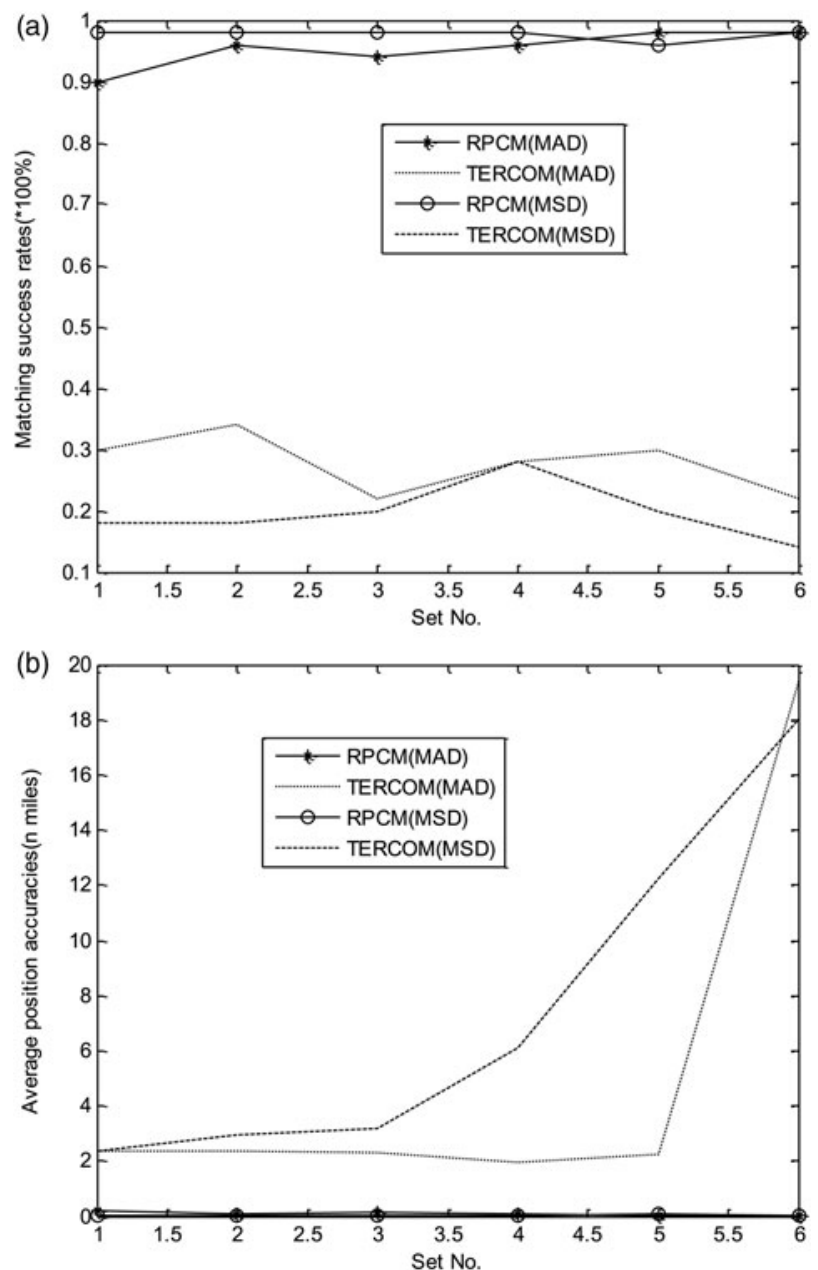

Figure 8. Curves of matching success rates and average position accuracies of RPCM and TERCOM. 

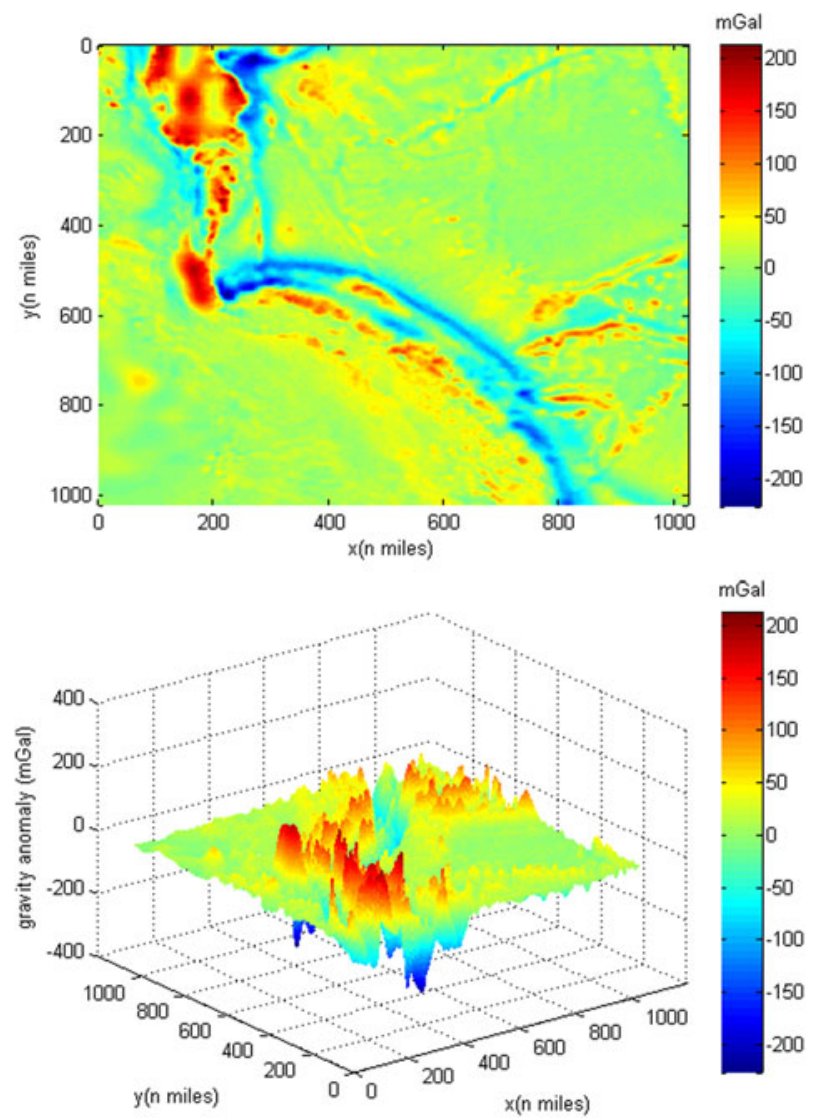

Figure 9. Gravity map $P_{2}$ located in the South China Sea.

Table 4. Statistical Values of Gravity Anomalies in gravity map $P_{2}$ (unit: $\mathrm{mGal}$ ).

\begin{tabular}{lcccc}
\hline Maximum & Minimum & Mean & STD & RMS \\
\hline $212 \cdot 35$ & $-227 \cdot 07$ & $8 \cdot 01$ & $41 \cdot 08$ & $41 \cdot 85$ \\
\hline
\end{tabular}

over the traditional TERCOM method are more obvious. Thus the shapes of trajectories are not restricted with the proposed RPCM method.

To verify the performance of the proposed RPCM method in different environments, another gravity anomaly map $P_{2}$ from a different area is taken to construct the same test. The size and resolution of the map $P_{2}$ are the same as the map $P_{1}$ but the features are quite different, as is illustrated in Figure 9 and the statistical values are given in Table 4.

Similarly, six sets of trajectories are simulated to test the RPCM and TERCOM method. The parameters are assigned as the same values as before. The resulting curves of matching success rates and average position accuracies are presented in Figure 10. Again, compared with TERCOM, obviously better matching success rates and position accuracies can be obtained by using the RPCM method. 

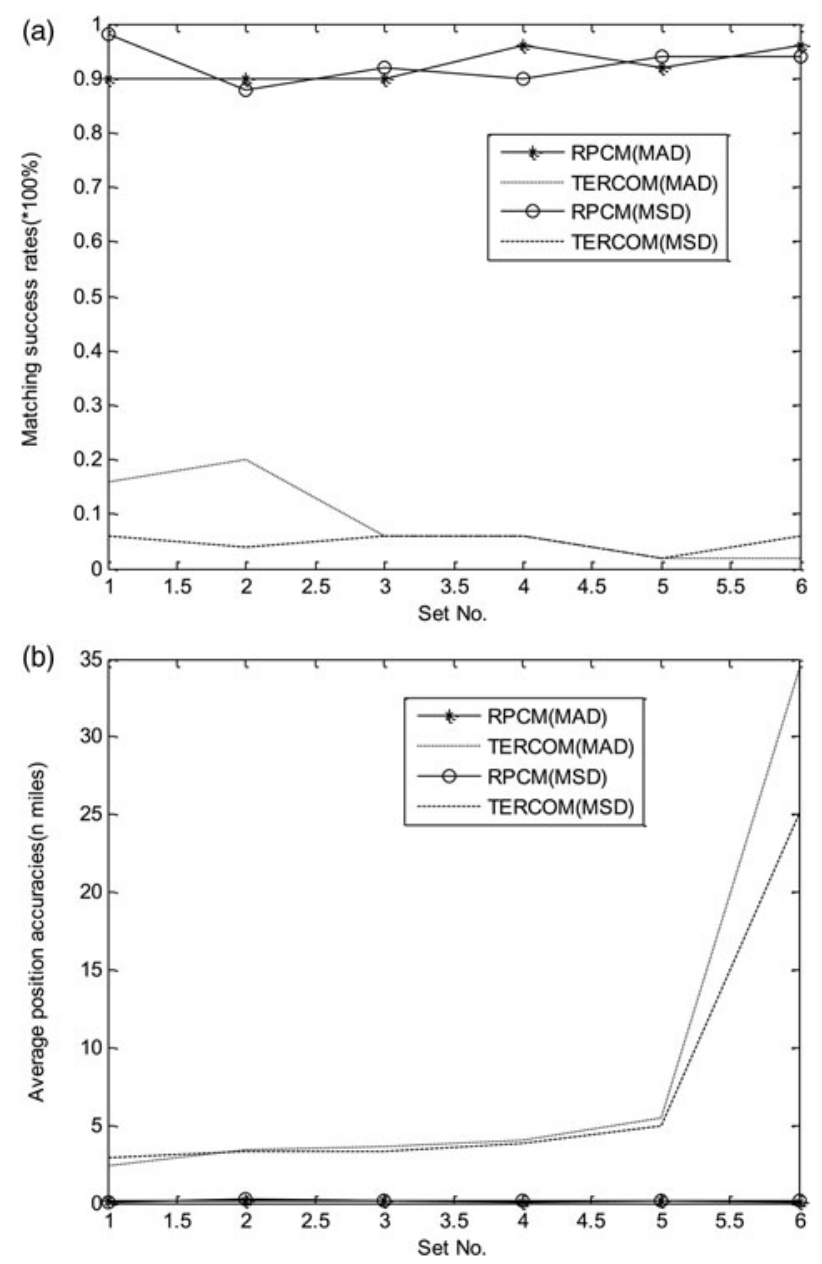

Figure 10. Curves of (a) matching success rates and (b) average position accuracies of RPCM and TERCOM (with gravity map $P_{2}$ ).

Therefore, with the higher performance in matching success rates and position accuracies, the proposed RPCM method should be preferable in practical navigation.

4. CONCLUSION. A Relative Positions-Constrained pattern Matching (RPCM) method for underwater gravity-aided inertial navigation is proposed in this paper. The gravity patterns are constructed based on the relative positions between points in a trajectory, which are calculated by INS indicated positions. In these patterns the accumulated position errors of INS are cancelled and removed. Thus the new constructed gravity patterns are more accurate and reliable, the process of matching can be constrained, and the probability of mismatching can also be reduced. Simulation results show that the performance, including matching success rate and position accuracy, is highly improved with the RPCM method. However, the effects of attitude or velocity errors cannot currently be entirely cancelled by using the proposed method. 
With the RPCM method the shapes of trajectories are not restricted as in traditional TERCOM methods so the novel method proposed in this paper is preferable in achieving the practical requirements of marine gravity-aided navigation. With this method the results of navigation may be in better agreement with the actual positions. Therefore, the accuracy and reliability of gravity-aided navigation would be improved.

In future research, more data from the true gravity sensors should be included in the simulations. The performance of gravity-aided inertial navigation in different environments (on surface or underwater) should also be investigated in a real marine test. Furthermore, present and future underwater INS/gravity integrated navigation systems would be supported and benefit from this research.

\section{ACKNOWLEDGEMENT}

The authors would like to thank the reviewers who provided comments that substantially improved this paper.

\section{FINANCIAL SUPPORT}

This research was supported by National Natural Science Foundation of China (Grant No. 41321063, 41104050, 41374086, 41204018 and 41274084), and Chinese Academy of Sciences International Partnership Program for Creative Research Teams (CAS/SAFEA) (Grant No. KZZD-EW-TZ-05).

\section{REFERENCES}

Affleck, C.A., and Jircitano, A. (1990). Passive gravity gradiometer navigation system. Proceedings of IEEE Position Location and Navigation Symposium, 60-66.

Ånonsen, K.B. (2005). Terrain aided AUV navigation - A comparision of the point mass filter and terrain contour matching algorithms. Proceedings of Undersea Defense Technology Europe 2005, Amsterdam, The Netherlands, June 21-23, 2005.

Ånonsen, K.B. (2010). Advances in terrain navigation for underwater vehicles. Doctoral thesis of Norwegian University of Science and Technology.

Bishop, G. (2002). Gravitational field maps and navigational errors. IEEE Journal of Oceanic Engineering, 27 (3), 726-737.

Cowie, M., Wilkinson, N., and Powlesland, R. (2008). Latest development of the TERPROM(R) Digital Terrain System (DTS). Proceedings of IEEE/ION Position Location and Navigation Symposium, 658-668.

Deng, Z., Ge, Y., Guan, W. and Han, K. (2010). Underwater map-matching aided inertial navigation system based on multi-geophysical information. Frontiers of Electrical and Electronic Engineering in China, 5(4), 496-500.

Golden, J. P. (1980). Terrain contour matching (TERCOM): A cruise missile guidance aid. in Wiener, T.F., ed. 'Image Processing for Missile Guidance', December 1980, The Society of Photo-Optical Instrumentation Engineers (SPIE), 238, 10-18.

Goldstein, M.S., and Brett, J.J. (1998). Precision gravity gradiometer/AUV system. Proceedings of The 1998 Workshop on Autonomous Underwater Vehicles, 167-174.

Hays, K.M. (2002). A submarine navigator for the 21st century. Proceedings of IEEE/ION Position Location and Navigation Symposium, 179-188.

Jekeli, C. (2006). Precision free-inertial navigation with gravity compensation by an onboard gradiometer. Journal of Guidance, Control and Dynamics, 29(3), 704-713.

Jircitano, A., White, J., and Dosch, D. (1990). Gravity based navigation of AUV's. Proceedings of the Symposium on Autonomous Underwater Vehicle Technology, 177-180. 
Kamgar-Parsi, B., and Kamgar-Parsi, B. (1999). Vehicle Localization on Gravity Maps. Proceedings of The SPIE Conference on Unmanned Ground Vehicle Technology, Vol. 3693, 182-191.

Moody, M.V., and Paik, H.J. (2004). A Superconducting Gravity Gradiometer for inertial navigation. Proceedings of IEEE/ION Position Location and Navigation Symposium, 775-781.

Moryl, J., Rice, H., and Shinners, S. (1998). The Universal Gravity Module for Enhanced Submarine Navigation. Proceedings of IEEE Position Location and Navigation Symposium, 324-331.

Rice, H., Kelmenson, S., and Mendelsohn, L. (2004). Geophysical navigation technologies and applications. Proceedings of IEEE Position Location and Navigation Symposium, 618-624.

Rice, H., Mendelsohn, L., and Aarons, R. (2000). Next generation marine precision navigation system. Proceedings of IEEE Position Location and Navigation Symposium, 200-206.

Siouris, G. M. (2004). Missile Guidance and Control System. Springer.

Vajda, S., and Zorn, A. (1998). Survey of existing and emerging technologies for strategic submarine navigation. Proceedings of IEEE Position Location and Navigation Symposium, 309-315.

Wang, H., Wang, Y., Fang, J., Chai, H., and Zheng, H. (2012). Simulation research on a minimum rootmean-square error rotation-fitting algorithm for gravity matching navigation. Science China-Earth Sciences, 55(1), 90-97.

Wang, S., Zhang, H., Yang, K., and Tian, C. (2010). Study on the underwater geomagnetic navigation based on the integration of TERCOM and K-means clustering algorithm. Proceedings of IEEE OCEANS 2010, $1-4$.

Wang, Z., and Bian, S. (2008). A local geopotential model for implementation of underwater passive navigation. Progress in Natural Science, 18(9), 1139-1145.

Wu, L., Gong, J., Cheng, H., Ma, J., and Tian, J. (2007). New method of underwater passive navigation based on gravity gradient. Proceedings of SPIE 5th International Symposium on Multispectral Image Processing and Pattern Recognition, p67901 V.

Wu, L., Ke, X., Hsu, H., Fang, J., Xiong, C., and Wang, Y. (2013). Joint gravity and gravity gradient inversion for subsurface object detection. IEEE Geoscience and Remote Sensing Letters, 10(4), 865-869.

Wu, L., Ma, J., and Tian, J. (2010a). A Self-adaptive Unscented Kalman Filtering for Underwater Gravity Aided Navigation. Proceeding of IEEE/ION Position Location and Navigation Symposium, 142-145.

Wu, L., and Tian, J. (2010) Automated Gravity Gradient Tensor Inversion for Underwater Object Detection. Journal of Geophysics and Engineering, 7(4), 410-416.

Wu, L., Tian, X., Ma, H., and Tian, J. (2011). A Matching-Unscented Kalman Filtering for Gravity Aided Navigation. Proceedings of SPIE 7th International Symposium on Multispectral Image Processing and Pattern Recognition, 80030P-80030P-5.

Wu, L., Tian, X., Ma, J., and Tian, J. (2010b). Underwater Object Detection Based on Gravity Gradient. IEEE Geoscience and Remote Sensing Letters, 7(2), 362-365.

$\mathrm{Xu}, \mathrm{D}$. (2005). Using gravity anomaly matching techniques to implement submarine navigation. Chinese Journal of Geophysics (in Chinese), 48(4), 812-816.

Yoo, Y.M., Lee, W.H., Lee, S.M., Park, C.G., and Kwon, J.H. (2012). Improvement of TERCOM aided inertial navigation system by velocity correction. Proceedings of IEEE/ION Position Location and Navigation Symposium, 1082-1087.

Zhang, F., Chen, X., Sun, M., Yan, M., and Yang, D. (2004a). Simulation design of underwater terrain matching navigation based on information fusion. Proceedings of IEEE International Geoscience and Remote Sensing Symposium, 3114-3117.

Zhang, F., Chen, X., Sun, M., Yan, M., and Yang, D. (2004b). Simulation study of underwater passive navigation system based on gravity gradient. Proceedings of IEEE International Geoscience and Remote Sensing Symposium, 3111-3113.

Zheng, H., Wang, H., Wu, L., Chai, H., and Wang, Y. (2013). Simulation Research on GravityGeomagnetism Combined Aided Underwater Navigation. Journal of Navigation, 66(1), 83-98. 